\title{
CUIDADOS PALIATIVOS: O PAPEL DA EQUIPE DE ENERMAGEM UM OLHAR DIFERENTE SOBRE AS NECESSIDADES ATUAIS
}

\author{
Sueli Barros ${ }^{1}$, Chennyfer Dobbins Abi Rached ${ }^{2}$ \\ 1 Graduanda de Enfermagem. Universidade Nove de Julho - UNINOVE. São Paulo - Brasil - \\ Email: São Paulo - Brasil-email: regiane0255@gmail.com \\ 2 Doutora em Saúde Coletiva; Mestre em Economia da Saúde pela Universidade Federal de São \\ Paulo (UNIFESP); Docente do Programa de Mestrado Profissional em Gestão em Sistemas de \\ Saúde - Universidade Nove de Julho - UNINOVE. São Paulo - Brasil email: \\ chennyferr@yahoo.com.br ORCID: https://orcid.org/0000-0002-4499-3716
}

\begin{abstract}
RESUMO
O tipo de assistência prestado para pacientes com doenças avançadas e terminais tem sido objeto de intenso debate na literatura especializada. Este artigo descreve e analisa, a partir de revisão de literatura, as intervenções possiveis no fim de vida, com especial ênfase aos cuidados paliativos, um modelo em expansão no mundo. Analise a partir de uma perpectiva bioética, alguns dos aspectos moralmente relevantes que envolvem estas práticas, que tendem a ser conflituosas entre as partes, especificamente, dentro do campo dos cuidados paliativos, destaca certas dificuldades e desafios em torno da centralidade da autonomia, considerada um dos pilares para boas práticas em cuidados no fim de vida.
\end{abstract}

Palavras Chaves: Cuidado Paliativo, Cuidados de Enfermagem, Humanização, Cuidados no fim de vida.

\begin{abstract}
The Type Of Assistance Provided For Patients With Diseases Advances Terminals Has Been Object Of Intense Debate In The Specialized Literature. This Article Describes Analysis From The Literature Review Of Interventions Possible At The End Of Life, With Special Reference To Physiological Care, A Model In Expansion In The World. Analysis From A Bioethics Perspective, Some Of The Aspects Morallly Relevant That Involves These Practices, Whichbetween The Parties, They Have To Be Conflited Between The Parties, Especially Within The Field Of Palliative Care, It Struggles Certain Difficulties And Challenges Around The Centrality Of Autonomy, Considering One Of The Pillars For Good Practices In Care At The End Of Life.
\end{abstract}

Key Words: Palliative Care, Nursing Care, Humanization, End-of-life Care 


\section{INTRODUÇÃO}

Termo Cuidados Paliativos é utilizado para expressar a ação de uma equipe multiprofissional a paciente fora de possibilidades terapêuticas de cura". A palavra "Paliativa "é originada do latim pallium que significa manto proteção, ou seja, proteger aqueles em que a medicina curativa não mais atende ${ }^{1}$. Diz o Manual dos Cuidados Paliativos que a origem do mesmo confunde - se historicamente com o termo "hospice ", abrigo que tinham de cuidar dos viajantes e peregrinos doentes, essas instituições eram mantidas por religiosos, cristãos dentro de uma perspectiva caridosa ${ }^{1}$.

Lidar com perdas onde um ambiente que visa cura e prevenção da doença predomina, é um desafio que poucos se propõe a discutir e menos ainda enfrentar, gerando dificuldade no tratamento e no acompanhamento dos pacientes com sofrimento intenso em fase terminal ${ }^{2}$.

Ajudar esses pacientes e seus familiares tem - se um modelo de cuidado chamado Cuidados Paliativos $^{2}$.

Os Cuidados Paliativos tiveram sua definição pela Organização Mundial de Saúde (OMS)1990 e redefinido em 2002 como sendo uma abordagem que aprimora a qualidade de vida, dos pacientes e familiares que enfrentam problemas associados com doenças, através prevenção, e alivio do sofrimento, por meio de identificação precoce, avaliação correta e tratamento da dor e outros problemas de ordem física, psicossocial e espiritual ${ }^{2}$.

Seus princípios incluem: reafirmar a importância da vida, considerando a morte como um processo natural, não a prolongando com medidas desproporcionais ${ }^{2}$.

A maioria dos profissionais da saúde que trabalham com pacientes terminais enfrentam desafios para tentar promover uma assistência de alta qualidade, sem esquecer do lado humano do cuidar ${ }^{3}$.

O respeito pelo outro em sua totalidade se constrói devagar, buscando uma relação de equilíbrio durante todo o processo do cuidar ${ }^{3}$.

Para os pacientes terminais precisa - se mais que técnica e sim percepções, com isso a percepção da morte na cultura ocidental é vista como perda, fracasso e, assim relacionada a sentimento de tristeza, medo e insegurança ${ }^{3}$.

\section{OBJETIVO}

Descrever o papel da equipe de enfermagem nos cuidados com os pacientes em Cuidados Paliativos. 


\section{METODOLOGIA}

O levantamento bibliográfico foi realizado pela internet em artigos indexados nos bancos de dados LILACS (Literatura Latino Americana em Ciências e Saúde), Scielo (Scientific Eletronic Library Online. Etapas seguidas: Leitura criteriosa desses dados, categorização dos assuntos e interpretação dos achados, as fontes pesquisadas variam de 2013 a 2017.

Descritores: Cuidado Paliativo, Cuidados de Enfermagem, Humanização, Cuidados no fim de vida.

\section{DESENVOLVIMENTO}

No decorrer dos anos, a percepção da morte foi se transformando, para os nossos antepassados a morte era percebida como uma fase natural da vida, o processo mortelmorrer era assistida pelos familiares ${ }^{4}$.

Houve uma transição de conceitos, uma morte que era consumada e constatada nas residências dos doentes, uma família que assumia os cuidados passa a transferir estes cuidados aos profissionais de saúde nas casas de saúde 4 .

Além disso, com a tecnologia na área da saúde proporcionou um aumento nas possibilidades terapêuticas fazendo com o que os profissionais conseguissem um certo controle sobre o tempo e as circunstancias da morte ${ }^{4}$.

Se de um lado a possibilidade terapêutica vem fazendo com o que novas formas de tratamento e esperança resolvam muitos problemas, estendendo os limites da vida a qualquer custo realizando tratamentos injustificáveis ${ }^{5}$.

Do outro lado trabalha - se com doenças sem prognostico, este é um trabalho que muitos não gostam de discutir, mais cuidar de indivíduos com doenças terminais é uma atividade ou modelo de atenção á saúde que vem sendo denominada Cuidados Paliativos ${ }^{5}$.

Em 1990 a Organização Mundial da Saúde definiu o conceito de Cuidados Paliativos e redefiniu em 2002, sendo cuidados ativos e totais do paciente cujo doença não responde mais ao tratamento curativo, a filosofia cuidados paliativos afirma a morte como um processo normal do viver, não apressa e nem adianta a morte, integra os aspectos psicológicos sociais e espirituais, disponibiliza uma rede de apoio ao paciente e seus familiares ${ }^{5}$.

A origem desse conceito foi na Inglaterra em 1967, com Cicely Saunders que fundou o primeiro "hospice", em Londres o Saint Christopher's Hospice que seria uma nova filosofia sobre a abordagem de pacientes terminais ${ }^{6}$.

No Brasil a história de Cuidados Paliativos tende a iniciar na década de 1980, em 1983 no Rio Grande do Sul, seguidos da Santa Casa de Misericórdia em São Paulo em 1986 e logo após em Santa Catarina e Paraná, um dos serviços que merece destaque é o Instituto Nacional do Câncer 
- INCA, que inaugurou em 1998 Hospital Unidade IV, exclusivamente dedicados ao Cuidados Paliativos $^{6}$.

A assistência de enfermagem em Cuidados Paliativos deve considerar o paciente um ser único, complexo e multidimensional: biológico, emocional, social, e espiritual. Este tipo de cuidado integral e humanizado, só é possível quando o enfermeiro faz uso de diversidades de comunicação para que perceba, compreenda e empregue a comunicação verbal e não verbal ${ }^{6}$. Porém a falta de habilidades e conhecimentos dos profissionais de enfermagem no que se refere a comunicação a pacientes sem possibilidades de cura dificulta esse trabalho de comunicação efetiva ${ }^{6}$.

Isso nos leva a pensar que por não conhecerem essas técnicas de comunicação terapêutica evitam o contato verbal com os pacientes que vivenciam o processo de morrer, afastando - se dos mesmos por não saber trabalhar sentimentos que a situação de morte iminente lhes desperta ${ }^{5}$.

Isso é preocupante pois os enfermeiros e sua equipe são os profissionais da área da saúde que interagem mais diretamente com o paciente durante sua estadia em uma instituição hospitalar ${ }^{6}$. Terminalidade tem como finalidade o cuidar que significa estar ao lado de pessoas fragilizadas com perda de vitalidade e autonomia, dor, depressão, o cuidado só é eficaz quando o ser cuidado - o paciente consente em ser alvo dessas ações aderindo ao plano assistencial proposto pelo profissional, o paciente só se permite ser cuidado quando se sente seguro e confia no profissional $^{6}$.

Em alguns relatos citados pelos pacientes podemos perceber que o diferencial é o olho no olho, são pequenos gestos como um olá, bom dia, boa noite, um sorriso, um abraço, uns apertos de mão são essenciais para que o paciente passe a confiar no profissional, isso faz com o que ele se sinta único, não se importando apenas com o que ele fala mais também com o que está sentindo e expressando ${ }^{6}$.

É muito natural que pacientes que vivenciam a terminalidade não gostem de falar sobre isso, e o profissional tem que respeitar suas vontades, e com isso os profissionais focam as conversas em outros assuntos que sejam prazerosos para o paciente trazendo assim um conforto na conversa ${ }^{7}$.

Em muitos relatos de pacientes em Cuidados Paliativos podemos perceber que a alegria dos profissionais, sempre sorrindo, incentivando ajudam muito no tratamento, porque aliviam a ansiedade, diminuem a angustia e muitas vezes faz até mesmo a esquecer por alguns momentos o sofrimento com frases de otimismo como: você vai melhorar, isso é passageiro, seja forte, a natureza alegre e otimista da interação enfermagem e paciente deve ser construída por ambas as partes, ambos visam manter o otimismo ${ }^{7}$. 
Manter o otimismo e a esperança não significa que iremos fingir ou mentir as reais preocupações e riscos dos pacientes e seus familiares, mais precisam ser seguidas e partilhadas do lado positivo da situação ${ }^{7}$.

A companhia, o estar junto também são formas de comunicação interpessoal, estar desacompanhado causa medo, os pacientes explicitam claramente em seus discursos o fato de sentirem - se mais confortáveis na presença de outras pessoas no momento da morte ${ }^{7}$.

Se não há o que dizer ou nada mais se pode fazer porque a morte é iminente, a presença compassiva, mesmo que silenciosa e a companhia que consola e conforta são maneiras sutis, mais de extrema importância e que será cuidado até o fim ${ }^{8}$.

As crenças religiosas e espirituais influenciam nas decisões e tratamento em situações de terminalidade ${ }^{8}$.

Os Cuidados Paliativos voltado a criança e adolescentes são definidos pela (Organização Mundial da Saúde em 2010 como uma modalidade de atenção que envolve o fornecimento de múltiplos suportes ao corpo físico, ao espirito e a mente do paciente e seus familiares, a (OMS) ainda preconiza que Cuidados Paliativos deve envolver um suporte multiprofissional á família em situação do luto antecipado, sendo a morte um processo natural do curso da vida ${ }^{8}$.

O câncer infantil é uma doença rara, mais nos últimos anos o câncer constitui maior causa a morte por crianças abaixo dos 15 anos de idade as neoplasias mais frequentes em crianças são: leucemias, linfomas 9 .

As crianças estabelecem vinculo e uma familiaridade com o ambiente hospitalar devido as internações recorrentes, isto faz com o que os profissionais da saúde desenvolvam vínculos e conheçam particularidade tanto da criança quanto da família, aprendendo a identificar suas necessidades para prestar cuidado com qualidade 9 .

Podemos observar em alguns artigos lidos que a equipe de enfermagem sofre mais com a morte da criança com câncer em Cuidados Paliativos, porque eles são vistos como se tem uma vida toda pela frente, muito jovem ${ }^{9}$.

Nesta condição chegam a questionar se fizeram tudo o que poderiam pela vida da criança, originando um sentimento de impotência e derrota em algumas situações ${ }^{9}$.

O profissional ao compartilhar o processo de morte investe todos os seus esforços para ajudar a familia9.

A equipe de enfermagem está mais próxima do paciente, por isso consegue prestar a assistência visando a qualidade de vida e a manutenção do conforto e operando as diversas tecnologias no auxílio das funções fisiológicas, a promoção do conforto, dentro das condições que a criança se encontra, é uma tarefa bastante especializada e requer da equipe de enfermagem mais atenção e habilidade 9 . 
Em uns dos depoimentos da enfermagem falaram que quando se trata de crianças eles fazem além dos cuidados básicos, além das condições fisiopatológicas para deixá-la a mais confortável possivel $^{9}$.

O profissional da equipe de enfermagem pode ser afetado pelo sofrimento dos pacientes e de seus familiares, envolvimento faz com o que os sentimentos dos pacientes se misture com suas próprias emoções, vivenciando assim o sofrimento e a dor sofrida pelo paciente, este envolvimento pode influenciar a atuação da equipe comprometendo os cuidados de enfermagem e levando os profissionais a sérios problemas entre eles: estresse, insatisfação, depressão provocando até mesmo um adoecimento ${ }^{10}$.

A equipe de enfermagem é a que mais permanece constantemente com os pacientes e familiares no decorrer das 24 horas $^{11}$.

A arte da enfermagem está em transformar um ser humano doente em um ser humano com melhor qualidade de vida, reconhecendo no processo de doença uma possibilidade de crescimento pessoal e social ${ }^{12}$.

Desse modo, representam a arte de cuidar através das palavras que afirmam o valor e a beleza da vida, como o amor, a estética, a identificação, o envolvimento, a vocação, a transformação, o despertar para a vida e ainda a linguagem dos sentidos; para eles são esses fenômenos que compõem a arte de cuidar ${ }^{13}$.

Os profissionais vivenciam um paradoxo de valores, porque de um lado se sentem aliviados, pelo termino do sofrimento do doente e do outro angustiam - se ante o quadro de dor que a morte traz contigo ${ }^{14}$.

O comprometimento de profissionais capacitados devidamente amparados por programa de sistema de saúde pode ajudar a alinhar as preferências dos pacientes e seus familiares com os planos de cuidado proporcionando mais qualidade de vida quando a cura se torna impossível. ${ }^{15}$. Para realizar um bom cuidado, a equipe de saúde necessita conhecer as técnicas, prestar informações, ter considerações para com o outro respeito postura e expressar interesse pelo que é dito pelo paciente e familiar ${ }^{16}$.

Nesse contexto o enfermeiro deve aperfeiçoar sempre suas habilidades em relação ao conhecimento técnico, cientifico e na capacidade de percepção das necessidades do paciente ${ }^{17}$. A uma necessidade de ter planejamento na assistência humanizada, pois o profissional de enfermagem é quem está mais próximo ao doente, o qual deve ser tratado de forma efetiva ${ }^{18}$. 


\section{CONSIDERAÇÕES FINAIS}

Podemos observar com este estudo que a importância da equipe de enfermagem em Cuidados Paliativos são além do que os próprios profissionais pensam, pois muitos dos pacientes em seus relatos falam que os gestos de carinho e atenção e dedicação que estes profissionais aplicam diminuem muito o sofrimento deles.

Os Cuidados Paliativos priorizam o quesito humanização e a relação saúde - paciente - família, proporciona uma resposta para as pessoas portadoras de doenças que ameaçam a continuidade da vida, desde diagnóstico da doença até seus momentos finais. A morte digna é de grande significado para o doente e também para o profissional que é compreensivo e solidário.

$\mathrm{O}$ antidoto para o sofrimento físico e espiritual do paciente em fase terminal é o conforto humano junto a familiares e entes queridos com a memória de um abraço e não de uma fria tecnologia.

\section{REFERÊNCIA BIBLIOGRAFICA}

1 Hermes HR, Lamarca ICA. Cuidados paliativos: uma abordagem a partir das categorias profissionais de saúde. Ciência e Saúde Coletiva vol. 18 no 9.Rio de Janeiro Sept.20013.

2.Monteiro FF, Oliveira M, Vall J. A importância dos cuidados paliativos na enfermagem.Rev Dor. São Paulo,2013 jul-set;11(3):242-248.

3.Santana JCB, Campos ACV, Barbosa BDG, Paula KF, Rezende MAE, Dutra BS. Cuidados Paliativos aos pacientes terminais percepção da equipe de enfermagem. Centro Universitário São Camilo - 2014;3(1):77 - 86.

4.Pinho IC,et al.As percepções do enfermeiro acerca da assistência. Rev Eletrônica Enf,2006 [citado 26 jul 2014];8(1):42-51.

5.Araújo MMT, Silva MJP.A comunicação com o paciente em cuidados paliativos: valorizando a alegria e o otimismo.Rev.esc.enferm.USP vol. 41 no.4 São Paulo Dec.2013.

6.Avanci BS, Carolindo FM, Góes GB, Netto NPC.Cuidados paliativos á criança oncológica na situação do viver\morrer:a ótica do cuidar em enfermagem.Esc Anna Nery vol13 no4 Rio de Janeiro Oct $\backslash$ Dec.2014.

7.Silva WCBP,Silva RMCR ,Pereira ER,Silva MA,Martins AMF,Sauthier M.Nursing team perception of oncological palliative care:a phenomenological study2014 Mar;13(1):72-81.

8.Moreira LM,Ferreira RA,Junior ALC.Discussão de protocolo para cuidadores de pacientes com câncer em cuidados paliativos. Paidéia (Ribeirão Preto) vol22 no 53 Ribeirão Preto Sept $\backslash$ Dec.2013.

9.Silva MM,Moreira MC.Sistematização da assistência de enfermagem em cuidados paliativos na oncologia:visão dos enfermeiros.Acta Paul Enferm 20115;24(2):172-178. 
10.Vargas MAO,Vivan J,Vieira RW,Mancia JR,Ramos FRS,Ferrazzo S,Bitencourt JVOV.Ressignificando o cuidado em uma unidade especializada em cuidados paliativos:uma realidade possível.Texto e Contexto enferm.vol,22 no3 Florianópolis July\Sept.2013.

11.Silva MM,Moreira MC,Leite JL, Erdmann AL.Análise do cuidado de enfermagem e da participação dos familiares na atenção paliativa oncológica.Texto e Contexto enferm vol.21no3 Florianópolis July\Sept.2016.

12.Sousa ATO, França JRFS,Santos MFO,Costa SFG,Souto CMRM.Cuidados paliativos com pacientes terminais:um enfoque na Bioética.Rev Cubana Enfermer,2015;26(3):123-35.

13.Foriani CA,Schramm FR,Desafios morais e operacionais da inclusão dos cuidados paliativos na rede de atenção básica.Cad Saúde Publica.[Internet].2016;23(9):2072-80.

14.Souza HL,Zoboli ELCP,Paz CRP, Schveitzer MC, Hohi KG, Pessalacia JDR.Cuidados Paliativos na atenção primária á saúde: considerações éticas.Rev. .Bioét.vol23 no2 Brasilia May\Aug.2015.

15.Araújo MMT, Silva MJP, Estratégias de comunicação utilizadas por profissionais de saúde na atenção a pacientes sob cuidados paliativos.Rev Esc Enferm USP.[internet\}.2012 [acesso 7 abr 2015];46(3): 626-32.

16.Becker AL,Ethical considerations of teaching spirituality in the academy.Nurs Ethics(online)2016.

17.Fioriani CA,Home based palliative care:challenges in the care of technology- dependente childen. J Pediatri 2018;86(1):15-20.

18.Santos DBA, Lattaro RCC,Almeida DA.Cuidados paliativos de enfermagem ao paciente oncológico terminal:Revisão da Literatura.Rev de Iniciação Cientifica da Libertas.São Sebastião do Paraiso,v.1,n.1,p.72-84,dez.2016. 